\title{
POLARIZATION OF INCLUSIVELY PRODUCED HYPERONS
} B. Lundberg, G. Bunce (a) R. Handler, R. Grobel(b)
R. March, P. Martin (c) L. Pondrom, M. Sheaff, C. Wilkinson

University of Wisconsin, Madison, wI 53706

P. T. $\operatorname{Cox}(d)$ J. Dworkin, E. C. Dukes, O. E. Overseth, P. Skubic(e)

University of Michigan, Ann Arbor, MI 48109

K. Heller, C. James

University of Minnesota, Minneapolis, MN 55455

A. Beretvas, L. Deck (f) T. Devlin, B. Edelman(g)

R. T. Edwards (h) R. B. Luk, J. Norem (i)

P. Petersen, R. Rameika (c) L. Schachinger $(j)$

G. Thomson, R. Whitman, P. Yamin (a)

Rutgers-The State University, Piscataway, NJ 08854

\section{Abstract}

We report here polarization results from a series of Fermilab experiments from the years 1974 through 1980 , with some preliminary data from a high $p$ polarization experiment completed in February 1982. The $\Lambda$ polarization has a remarkably simple and interesting behavior when expressed as a function of $x_{F}$ and $\mathrm{p}_{\mathrm{T}}$.

The discovery of $\Lambda$ polarization from $300 \mathrm{GeV}$ inclusive proton-nucleon reactions ${ }^{1}$ initiated a series of successful experiments at Fermilab which measured polarizations and magnetic moments for $\Lambda, \Xi 0, \Xi^{-}, \Sigma^{+}$, and $\Sigma^{-}$hyperons ${ }^{2,3,4}$ The $\Lambda$ data is by far the most extensive and well studied with over $10^{7}$ events reconstucted to date at many production angles. Hence for the $\Lambda$ 's a detailed behavior of inclusive polarization with respect to the kinematic variables can be determined from the data.

The experimental apparatus is described in detail in References 2 and 3. Briefly, it consists of a system of magnets to pitch the proton beam on target at a specific production angle, followed by a collimation system imbedded in a primary beam dump magnet which defines the secondary hyperon beam. The hyperons are detected by a conventional proportional chamber spectrometer. For the high $\mathrm{p}_{\mathrm{T}}$ experiment, the production angle, $\theta_{\mathrm{p}}$, could be varied from 0 to +25 milliradians. Most of the data was taken with $\theta_{\mathrm{p}}$ set to $+20,+12,+10$, and +6 milliradians, although only 20 
and 12 milliradian data is presented here. For all results given here the reaction is

$$
\mathrm{p}+\mathrm{A} \rightarrow \mathrm{h}+\mathrm{X}
$$

where the target, $A$, is hydrogen, beryllium, copper, or lead, and $h$ is the particular hyperon being studied.

The behavior of $\Lambda$ polarization as a function of $\mathrm{p}_{\mathrm{T}}\left(\mathrm{p}_{\mathrm{T}} \leqslant 2 \mathrm{GeV}\right)$ for fixed production angle, $\theta_{p^{\prime}}$ is now well known. The magnitude of the polarization, $|P|$, increases monotonically with $\mathrm{p}_{\mathrm{T}}$. This trend, in itself, is not very illuminating since the important scaling variable $x_{F}$ is not fixed for constant $\theta_{p}$. To map out the polarization as a function of both $\mathrm{p}_{\mathrm{T}}$ and $x_{F}$ one needs to have many sets of data with each at a different production angle. A large sample of $\Lambda$ 's with 9 different values of $\theta_{p}$ has already been studied and reported 5,6 . This data and the preliminary results from the latest higher $\mathrm{p}_{\mathrm{T}}$ experiment, are compltetely consistent and support the observations given below.

The magnitude of the $\Lambda$ polarization, $|P|$, as a function of $x_{F}$ for three ranges of $p_{T}$ is shown in Figure 1. A linear fit to the data points constrained to pass through the origin is indicated with the determined slope. The behavior can be written

$$
|P|=C\left(p_{T}\right) x_{F}
$$

where $C\left(p_{T}\right)$, the slope, depends only on the transverse momentum.

The same data as in Figure 1 is also shown in Figure 2, however the polarization is plotted as a function of $p_{T}$ for four bins of $x_{F}$. The most noteworthy feature is that $|P|$ is independent of $p_{T}$ for $\mathrm{p}_{\mathrm{T}} \approx 1 \mathrm{GeV} / \mathrm{c}$. In addition, the value of $|\mathrm{P}|$ at which the polarization "saturates", i.e. $\mathrm{p}_{\mathrm{T}}>1 \mathrm{GeV} / \mathrm{c}$, increases linearly with $x_{F}$, as shown in Figure 3 . The preliminary data points are indicated by the open circles and show the highest $\mathrm{p}_{\mathrm{T}}, 3.4 \mathrm{GeV} / \mathrm{c}$, that can be attained with the present (incomplete) data sample. Once the data analysis is complete a measurement of polarization near $4 \mathrm{GeV} / \mathrm{C}$ will be possible.

It must be pointed out that no distinction has been made between directly produced $\Lambda$ 's and the daughter $\Lambda^{\prime} s$ from $\Sigma^{\circ}$, $\Sigma^{\circ}$ or other resonance decays. The polarization of the $\Sigma^{O}$ is not yet known and hence the measured $\Lambda$ polarization is diluted by some undetermined factor. This factor, proportional to the ratio of $\Lambda$ and $\Sigma^{\circ}$ cross sections, should not have a strong $\mathrm{p}_{\mathrm{T}}$ dependence, hence daughter $\Lambda$ contamination is not beleived to have much effect on the qualitative behavior described above. 
The data from Reference 3 is also shown on Figures $1 \& 2$ to illustrate the similarity of the results. The $\Xi 0$ data includes three production angles, and so offers the second best independent polarization sample for mapping out the $x_{\mathrm{F}}$ and $\mathrm{p}_{\mathrm{T}}$ trends.

The charged hyperon polarizations show the same qualitative trend as the $\Lambda^{\prime}$ s but since there is far less data taken at different production angles it is hard to draw the same conclusions as in the data. The results of the polarization analyses for $\Xi^{-}, \Sigma^{+}$, $\Sigma^{-}$, are shown in Figure 4 , at fixed $\theta_{\mathrm{p}}$ instead of fixed $x_{F}$. For comparison, the $\Lambda$ polarization at $\theta_{p}=5$ miliradians is shown by the dashed line. The sign is important: both $\Sigma^{+}$, and $\Sigma^{-}$are polarized in the opposite sense relative to the $\Lambda, \Xi^{0}$, and $\Xi^{-}$. The data for both the $\Sigma^{*}$ and $\Sigma^{-}$was obtained with only one value for the magnetic field in the precession magnet which implies that the sign of the polarization cannot be unambiguously determined without some other reference. For the $\Sigma^{+}$, the magnetic moment is known well enough to constrain the initial polarization ${ }^{7}$, and for the $\Sigma^{-}$the preliminary data from the Charged Hyperon Group at Fermilab provides the necessary constraint 8.9 .

One must remember, though, that polarization is not a universal feature of inclusive hyperon production. Data for $T^{\prime}$ s produced from a proton beam shows no measurable signal at $\mathrm{p}_{\mathrm{T}} \leq 1 \mathrm{GeV} / \mathrm{c}$ and preliminary results at higher $\mathrm{p}_{T}$ also show $\mid \mathrm{P} \Pi^{\prime}$ consistent with zero up to $\mathrm{p}_{\mathrm{T}} \approx 2.2 \mathrm{GeV} / \mathrm{C}$. Another interesting feature of polarization is that it appears to be independent of target material at Fermilab energies $(\sqrt{\mathrm{s}}=27 \mathrm{GeV})$. However, polarization data at lower energy $(\sqrt{5}=5$ $\mathrm{GeV}$ ) seems to show a strong dependence on target material. There does, however, seem to be a strong dependence on target material at lower energies 10

\section{Conclusion}

The previously analyzed data and the preliminary data from the higher $p_{T}$ polarization experiment are consistent with each other and the properties of inclusive polarization can be summarized with these important points:

(1) For inclusive $\Lambda$ polarization the $x_{F}$ behavior is striking and can be expressed as

$$
|P|=C\left(p_{T}\right) x
$$

(2) $\Lambda$ inclusive polarization becomes essentially independent of $p_{T}$ for $p_{T} \geqslant 1 \mathrm{GeV} / \mathrm{c}$. 
(3) Charged hyperons show the same qualitative polarization behavior as $\Lambda$ 's and $\Xi^{\text {ot }} s$.

(4) $\bar{\Lambda}$ 's show no measurable polarization for $p_{T}$ up to $2.2 \mathrm{GeV} / \mathrm{c}$ (preliminary result).

Present addresses :

(a) Brookhaven National Lab, Upton, NY 11973

(b) Salomon Brothers Inc., N. Y., NY 10004.

(c) Fermilab, Batavia, IL 60510.

(d) EP Division, CERN, CH-1211 Geneva 23, Switzerland.

(e) University of Oklahoma, Norman, OK 73069

(f) Max Planck Institut fur Physik und Astrophysik, 8 Munchen 23, B. R. D.

(g) Ford Motor Company, Allen Park, MI 48101.

(h) Bell Labs, Holmdel, NJ 07733

(i) Argonne National Lab, Argonne, IL 60439.

(j) Bell Labs, Murray Hill, NJ 07974.

l. G. Bunce et al., Phys. Rev. Lett. 36, 1113 (1976).

2. 0 . Overseth, Proceedings of the Fourth International Symposium on High Energy Spin Physics, Lauzanne, Switzerland, 1980 .

3. C. Wilkinson et al., Phys. Rev. Lett. 46, 803 (1981)

4. K. Heller et al., Phys. Rev. Lett. 41, 607

(1978). Grobel, Ph.D. Thesis, University of Wisconsin, 1980 (unpublished).

6. $K$. Heller, Proceedings of the Twentieth

International Conference on High Energy Physics, Madison, Wisconsin, 1980.

7. R. Settles et al., Phys. Rev. D 20, 2154 (1979).

8. L. Pondrom, contributed paper to this conference.

9. P. Cooper, contributed paper to this conference. 10. K. Takikawa, contributed paper to this conference. 

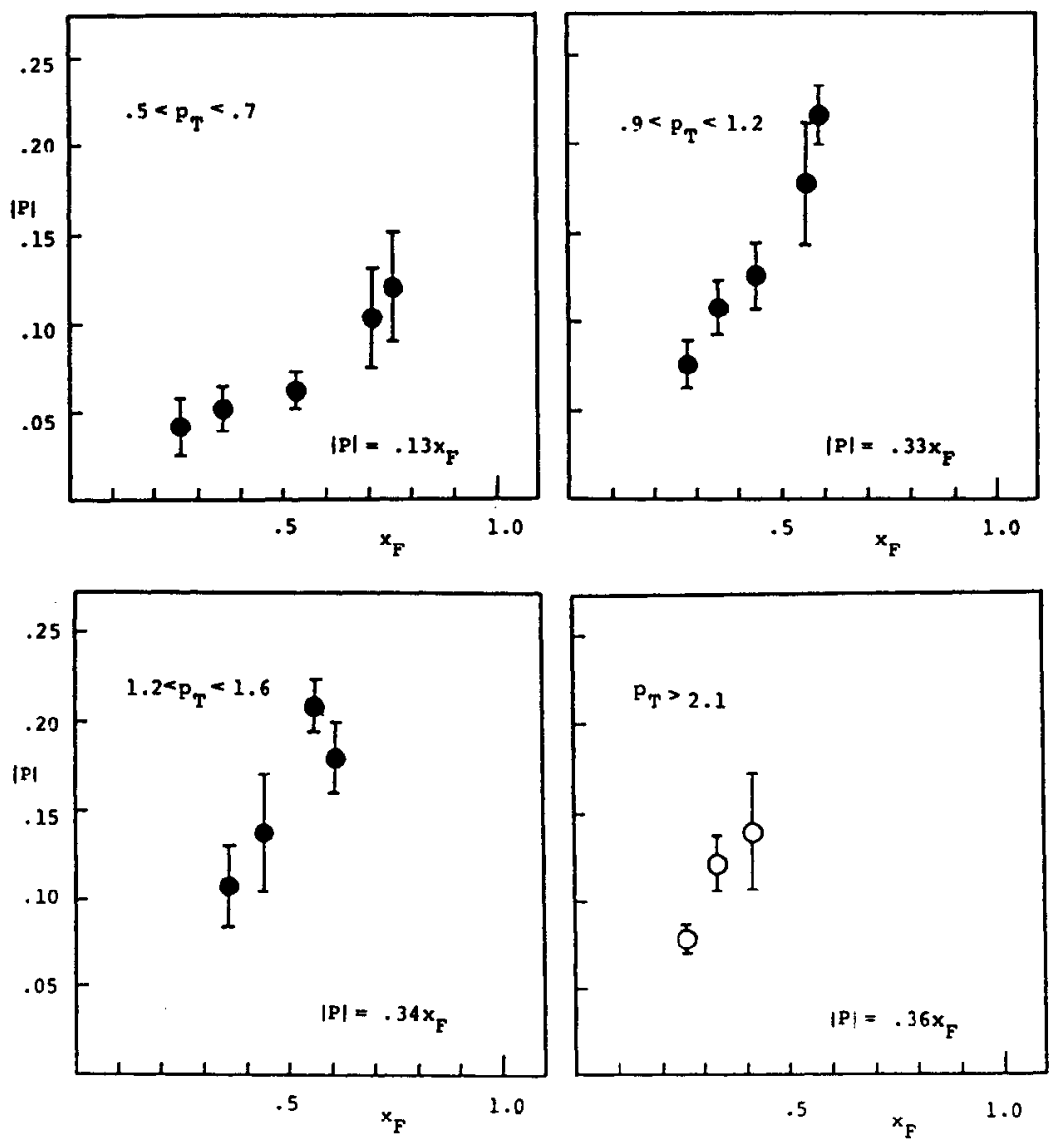

Fig. 1. The magnitude of inclusive $\Lambda^{\circ}$ polarization, $|P|$, as a function of $x_{F}$ for different bins of $\mathrm{p}_{\mathrm{T}}$ (in $\mathrm{GeV} / \mathrm{c}$ ). The open circles are based on preliminary data. A Iinear fit to the data points constrained to go through the origin was made for each $\mathrm{p}_{\mathrm{T}}$ range and is indicated on the graphs. 


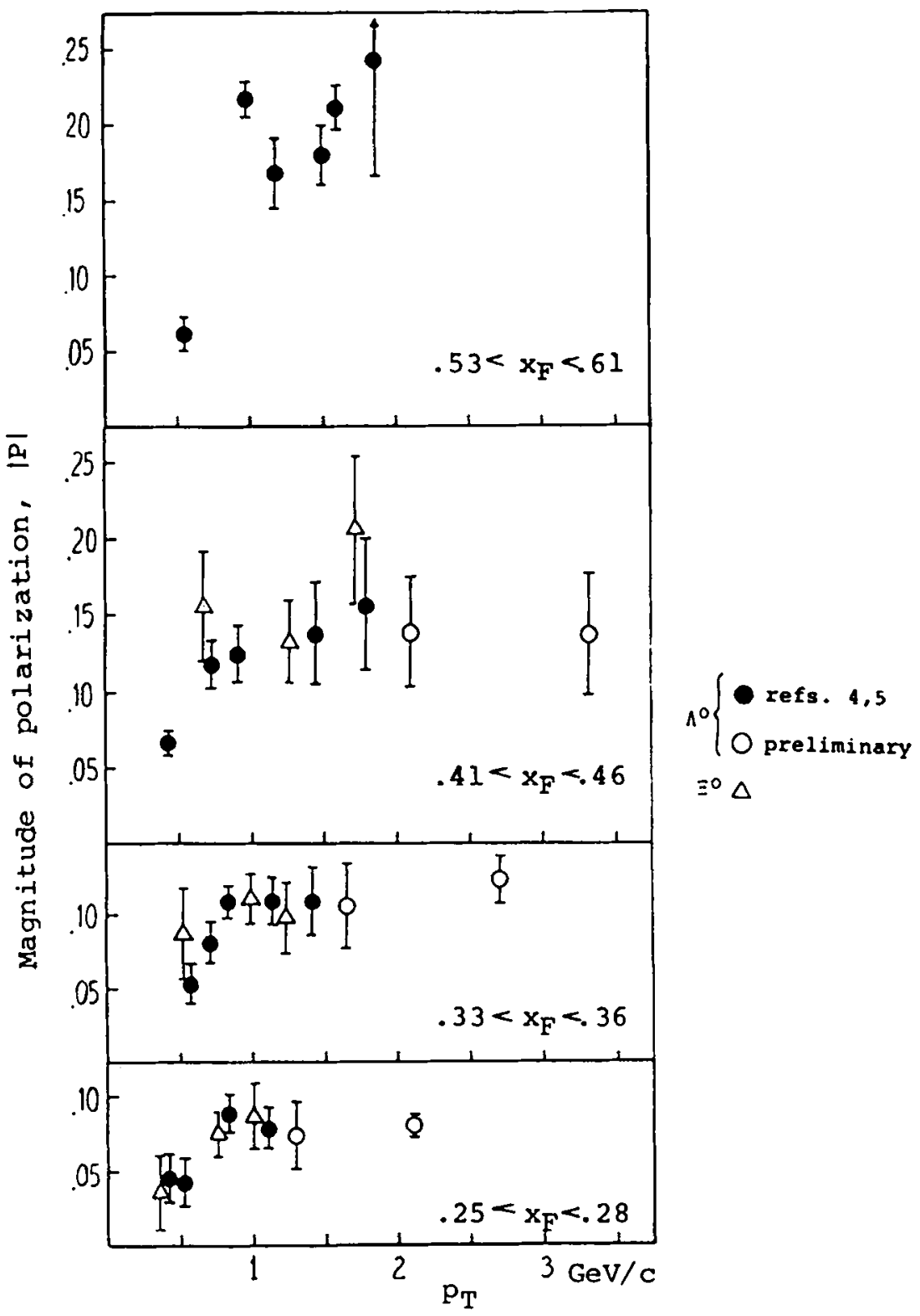

Fig. 2. The magnitude of inclusive $\Lambda^{\circ}$ and $\Xi^{\circ}$ polarization, $|\mathrm{P}|$, is shown as a function of $\mathrm{P}_{\mathrm{T}}$ for a fixed range of $x_{F}$. For $\Lambda^{\circ} \mathrm{s}$ it is clear that the polarization "saturates" for $\mathrm{p}_{\mathrm{T}} \geq 1 \mathrm{GeV} / \mathrm{c}$. 


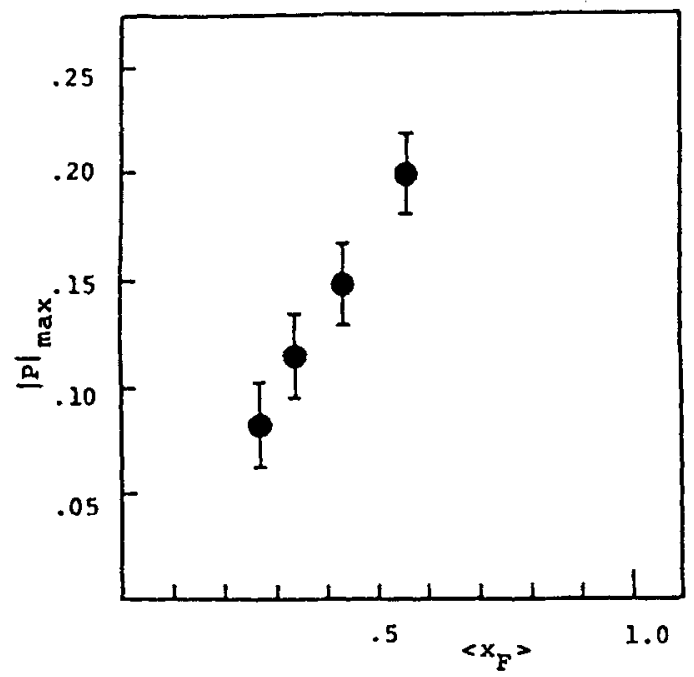

Fig. 3. The value of the maximum or "saturated" polarization for each $\mathrm{x}_{\mathrm{F}}$ range is determined by the mean value of $|\mathrm{P}|$ for those data points in Fig. 2 with $p_{\mathrm{T}}>1 \mathrm{GeV} / \mathrm{c}$. This maximum value, $|P| \max$ is plotted as a function of the mean value of $x_{F}$.

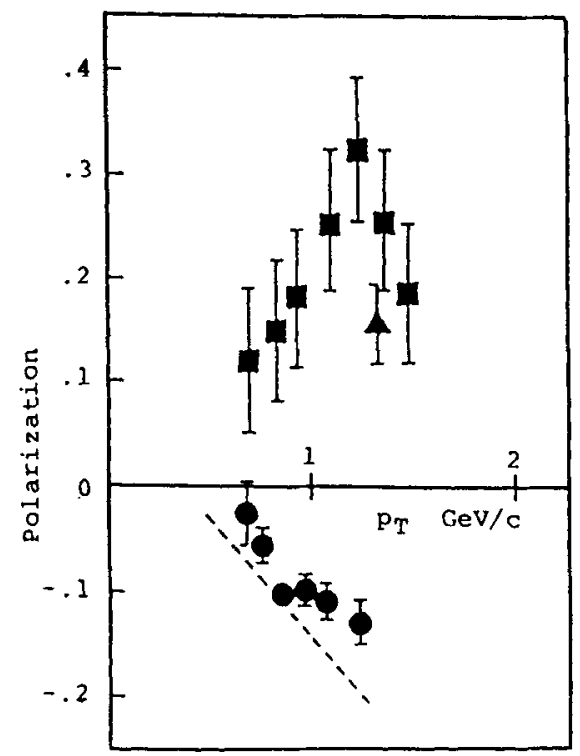

- $\Xi^{-} \theta_{\mathrm{p}}=5 \mathrm{mrad}$

- $\Sigma^{+} \theta_{p}=5 \mathrm{mrad}$

$\Delta \Sigma^{-} \theta_{\mathrm{p}}=7.5 \mathrm{mrad}$

… $\wedge^{\circ} \theta_{\mathrm{p}}=5 \mathrm{mrad}$

Fig. 4. Polarization of the charged hyperons for fixed production angle, $\theta_{p}$. For comparison a linear fit to the $\Lambda^{\circ}$ polarization at $\theta_{p}=5$ milliradians is also shown. 